\title{
Reconfigurable Partially Reflective Surface Antennas
}

\author{
Pei-Yuan Qin ${ }^{1} \quad$ Y. Jay Guo ${ }^{1} \quad$ Lu-Yang $\mathbf{J i}^{2}$ \\ (Global Big Data Technologies Centre, University of Technology Sydney, NSW, Australia) ${ }^{1}$ \\ (School of Electronics and Information, Northwestern Polytechnical University, Xi' an 710129)2
}

\begin{abstract}
In this paper, the research of reconfigurable partially reflective surface (PRS) antennas at University of Technology Sydney (UTS) is introduced. Two reconfigurable PRS antennas are described that can achieve beam scanning and wideband polarization switch, respectively.
\end{abstract}

Keywords: Reconfigurable antennas; beam scanning; polarization switch; partially reflective surface antenna.

\section{Introduction}

Reconfigurable antennas have received considerable attention from both academia and industry due to their capability of providing more functionalities than traditional antennas for applications in the advanced wireless communication systems [1]. However, most of the reconfigurable antennas suffer from low realized gains.

Partially Reflective Surface (PRS) antenna [2-4] provides a new way to realize high realized gains. It usually employs a microstrip antenna acting as the radiating source and a metallic or metallo - dielectric periodic array, located approximately half a wavelength above the radiator to enhance the directivity. Recently, reconfigurable PRS antennas have been developed to dynamically change the characteristics of the antenna, in terms of the operating frequency [2], polarization [3], and the radiation pattern [4].

This paper introduces two kinds of reconfigurable PRS antennas proposed by our group. Section II introduces a beam steering pattern reconfigurable PRS antenna which employs a new nonuniform reconfigurable PRS structure and a two-element microstrip patch phased array as the source. The antenna can switch its beam between $-15^{\circ}, 0^{\circ}$, and $15^{\circ}$ in an overlapped frequency range that goes from 5.5 to $5.7 \mathrm{GHz}$ (4\% impedance bandwidth) with a realized gain over $10 \mathrm{dBi}$ [5]. In Section III, a wideband polarization reconfigurable PRS Antenna is presented which employs a shorted annular patch (SAP) antenna as the source and a reconfigurable Wilkinson power divider network. It can switch its polarization among LP, RHCP, and LHCP in an overlapped bandwidth of 4.7-5.36 $\mathrm{GHz}(13.1 \%)$ with an average realized gain around $9 \mathrm{dBi} / \mathrm{dBic}[6]$.

\section{Pattern reconfigurable PRS antenna}

A reconfigurable PRS antenna with a beam-switching capability was proposed by our research group [5]. The beam scanning is realized by employing a reconfigurable PRS structure and a phased array as the source. Its beam can be switched between $-15^{\circ}, 0^{\circ}$, to $15^{\circ}$ with respect to the broadside direction from $5.5 \mathrm{GHz}$ to $5.7 \mathrm{GHz}$. The realized gains are over $10 \mathrm{dBi}$.

The overall structure of the proposed pattern reconfigurable PRS antenna is shown in Fig.1 (a). It consists of a two-element microstrip patch antenna and a reconfigurable feed network as the source, and a reconfigurable PRS structure. Fig 1. (b) and (c) give the details of array source and the reconfigurable PRS. 


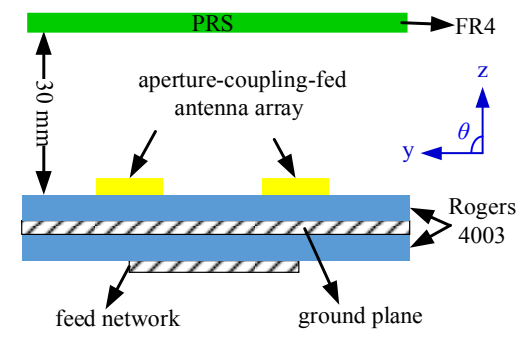

(a)

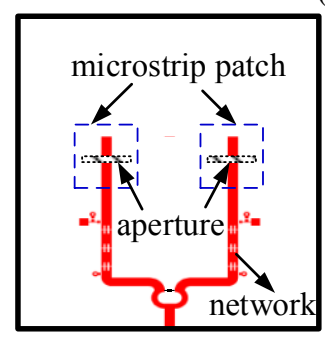

(b)

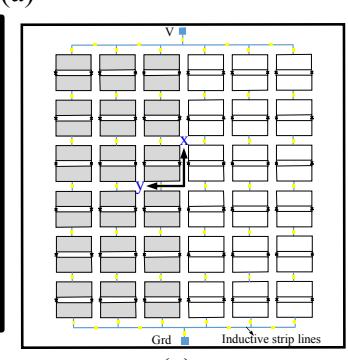

(c)
Fig. 1. Phased array fed PRS antenna (a) side view, (b) array source, and (c) reconfigurable PRS

The phased array source is comprised of two square microstrip patches. They are fed by microstrip lines printed on the other side of the substrate through coupling aperture. The reconfigurable feed network is constructed from Wilkinson power divider with Defected Microstrip Structure (DMS). For the PRS structure, it consists of $6 \times 6$ reconfigurable cells printed on the lower side of an FR4 substrate. The dimensions of the antenna are $150 \mathrm{~mm} \times 150 \mathrm{~mm}\left(2.85 \lambda_{0}\right.$ at $\left.5.7 \mathrm{GHz}\right)$.

Fig. 2 shows the simulated and measured results of the radiation patterns at $5.7 \mathrm{GHz}$. Its beam direction can be tilted between $-15^{\circ}$, $0^{\circ}$, and $15^{\circ}$ with a realized gain over $10 \mathrm{dBi}$. For the three scanning states, an overlapped impedance bandwidth from $5.5 \mathrm{GHz}$ to 5.74 $\mathrm{GHz}$ is achieved. The input reflection coefficients are not shown due to the limited space.

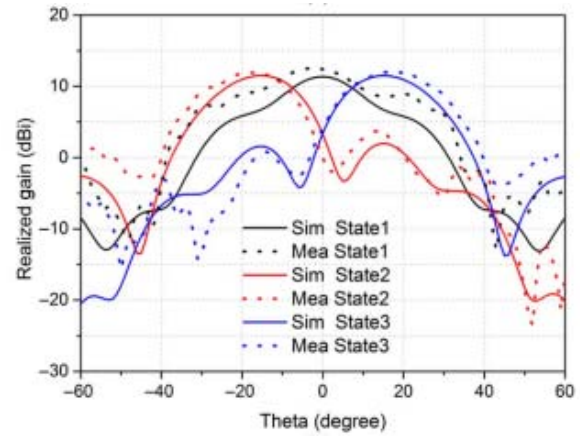

Fig. 2. Radiation patterns of the proposed antenna at $5.5 \mathrm{GHz}$.

Compared to some state-of-the-art pattern reconfigurable PRS antennas, the proposed antenna exploits two reconfiguration mechanisms to achieve a larger beam tilt angle without sacrificing the realized gains.

\section{Polarization reconfigurable PRS antenna}

Polarization switch between LP and CP can enable the antenna to adapt to different electromagnetic environments. However, due to their different input impedances, it is difficult to achieve a good impedance match for LP and CP simultaneously.

Our group proposed a polarization reconfigurable PRS antenna which can effectively solve the above problem [6]. It consists of a shorted annular patch (SAP) antenna as the source, a partially reflective surface (PRS) structure to enhance the gain, and a reconfigurable Wilkinson power divider as the feed network. The antenna can electronically alter its polarization between linear polarization (LP), left-hand circular polarization (LHCP), and right-hand circular polarization (RHCP), achieving an overlapped 10-dB impedance bandwidth and 3-dB axial-ratio bandwidth of $4.68-5.33 \mathrm{GHz}$ (13\%).

Fig. 3 shows the configuration of the proposed polarization reconfigurable PRS antenna. The PRS consists of an array of $5 \times 5$ square metallic patches (SMP). The source is a two-layer SAP structure fed by two probes. The details of the reconfigurable feed network are given in Fig.4. By controlling the states of the PIN diodes inserted in the power divider, the phase shift between Port 2 and Port 3 can be changed, thus leading to the polarization switch.

Fig. 5 gives the simulated and measured axial ratios for the $\mathrm{CP}$ modes. It can be seen that from $4.7 \mathrm{GHz}$ to $5.38 \mathrm{GHz}(13.5 \%)$ the measured axial ratio is below $3 \mathrm{~dB}$ for both LHCP and RHCP. The overlapped $10 \mathrm{~dB}$ impedance bandwidth and $3 \mathrm{~dB}$ AR bandwidth ranges from $4.7 \mathrm{GHz}$ to $5.36 \mathrm{GHz}(13.1 \%)$ for the three polarization modes. 


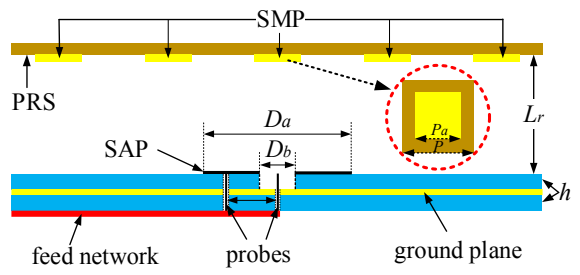

Fig.3 Structure of the polarization reconfigurable antenna

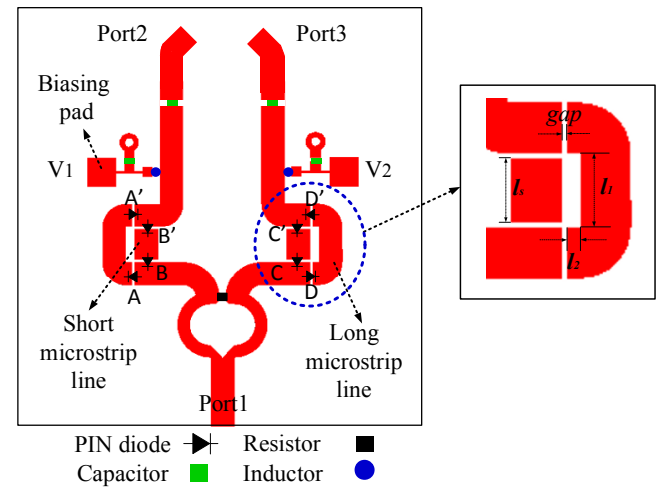

Fig.4 The configuration of the proposed feed network

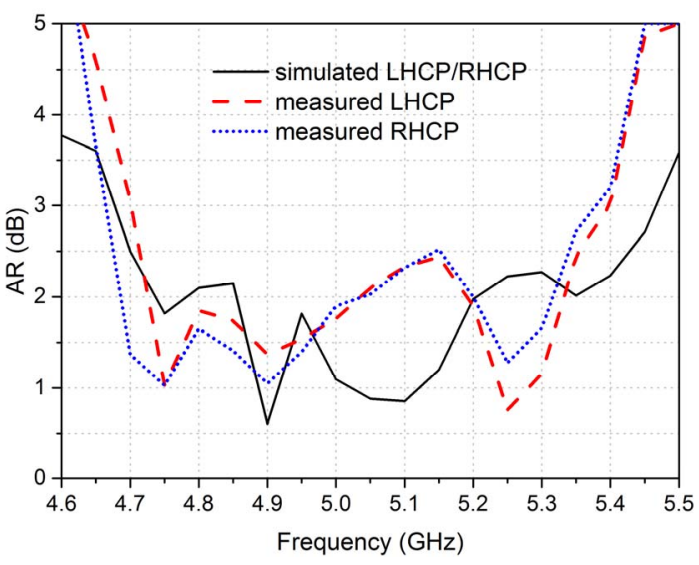

Fig.5 Axial ratio of the proposed antenna

The proposed antenna outperforms most of the reported polarization reconfigurable antennas for its wide bandwidth and relatively high gains, which makes it a promising candidate for future wireless communication systems.

\section{Conclusion}

In this paper, two reconfigurable PRS antenna have been introduced. The beam-scanning pattern reconfigurable one can switch its beam in the range of $\pm 15^{\circ}$ by employing a reconfigurable PRS structure and a phased array source. The polarization reconfigurable one can switch its state among LP, RHCP, and LHCP in a relatively wide operating bandwidth by using a reconfigurable feed network.

\section{Reference}

1 P. Y. Qin, Y. J. Guo, Y. Cai, E. Dutkiewicz, and C. H. Liang, A Reconfigurable Antenna With Frequency and Polarization Agility, IEEE antennas and wireless propagation letters, 2011, 10: 1373-1376.

2 A. R. Weily, T. S. Bird, and Y. J. Guo, "A reconfigurable high-gain partially reflecting surface antenna, IEEE Trans. Antenna Propag., vol. 56, no. 11, pp. 3382-3390, Nov. 2008.

3 H. L. Zhu, S. W. Cheung, X. H. Liu, and T. I. Yuk, Design of polarization reconfigurable antenna using metasurface, IEEE Trans. Antenna Propag., 2014, 62(6): 2891-2898.

4 T. Debogovic, J. Perruisseau-Carrier, Array-fed partially reflective surface antenna with independent scanning and beamwidth dynamic control, IEEE Trans. Antenna Propag., 2014, 62 (1): 446-449

5 L. Y. Ji, Y. J. Guo, P. Y. Qin, S. X. Gong, R. Mittra, A Reconfigurable Partially Reflective Surface (PRS) Antenna for Beam Steering, IEEE Trans. Antenna Propag., 2015, 63(6): 2387-2395.

6 L. Y. Ji, P. Y. Qin, Y. J. Guo, C. Ding, G. Fu, A Wideband Polarization Reconfigurable Partially Reflective Surface Antenna, IEEE Trans. Antenna Propag., 2016, 64(10): 4534-4538. 